\title{
Scintigraphy for Pulmonary Embolism: Too Old to Rock 'n' Roll, Too Young to Die?
}

$\mathbf{I}_{\mathbf{n}}$ lung scintigraphy is not only safe but also accurate enough to be quite useful clinically in patients with suspected acute pulmonary embolism (PE). However, many practitioners abandoned scintigraphy after the advent of CT pulmonary angiography, and scintigraphy remains underused in most medical centers. What can be done to harmonize clinical practice with recent data? The article by Glaser et al. in this issue (1) provides some pragmatic answers and supports the conclusion that scintigraphy is a reliable and clinically relevant test for PE.

See page 1508

The best way to assess diagnostic tests is to perform diagnostic accuracy studies that evaluate test performance objectively in a sample of patients pertinent to clinical situations. The STARD criteria provide a summary guideline for valid studies (2). Once the operating characteristics of the test are established, likelihood ratios and prior probability (odds) estimates provide diagnostic posterior probability (odds). Rational risk-benefit analyses of further evaluation and treatment options are then possible. Unfortunately, this preaching is rarely practiced in the trenches. Data often are

Received May 18, 2011; revision accepted May 26, 2011.

For correspondence or reprints contact: Henry Dirk Sostman, Methodist Hospital Weill Cornell, 6565 Fannin St., Ste. Dunn 200, Houston, TX 77030.

E-mail: dsostman@tmhs.org

Published online Jul. 27, 2011.

COPYRIGHT (c) 2011 by the Society of Nuclear Medicine, Inc.

DOI: 10.2967/jnumed.111.091744 not available or not applicable to specific patients. The parameters needed for risk-benefit analysis usually are not known numerically-or at all. The clinical setting does not tolerate ambiguity gracefully. How much more beguiling to the clinician than a probability scale and reference to Bayes' theorem is a crisp answer of "positive" or "negative," particularly when accompanied by an anatomically intuitive image of a blood vessel? However, the article by Glaser et al. shows that scintigraphy can play in this league safely, effectively, and with referring clinician concurrence.

Based on the mindset of radioimmunoassay, the venerable probability scale approach to scintigraphic diagnosis of PE has never met with understanding by referring clinicians (3). The trinary interpretation approach tested by Glaser et al. evolved more recently and has 2 key components: clear-cut "positive" and "negative" categories and infrequent recourse to a "nondiagnostic" category. Work to reduce the nondiagnostic category was critical to the clinical feasibility of the trinary approach. Several studies demonstrated that normal, nearnormal, very low probability, and low-probability scintigrams could be classified safely as negative (4-6). Work by the PIOPED investigators (7) and the PISAPED group (8) supported the judgment of Glaser et al. that single mismatched vascular defects can be classified as positive. Recent diagnostic accuracy studies of trinary interpretation showed good to excellent sensitivity and specificity $(9,10)$. By synthesizing these prior data into a practical clinical method, Glaser et al. were able to reduce the rate of nondiagnostic interpretations to $5 \%$ or less-compared with a recent multicenter outcome study in which $54 \%$ of scintigrams were classified as nondiagnostic (11)—with satisfactory patient outcomes. Glaser et al. have made an important contribution by demonstrating this prospectively in the clinical setting.

Other, more generic issues also are pertinent to this study.

Previous diagnostic accuracy studies of trinary classification have shown good but imperfect sensitivity and specificity $(9,10)$. Are those results consistent with the benign patient outcomes reported by Glaser et al.? We must recognize that clinical outcome studies of PE, although clinically relevant, provide an optimistic assessment of negative predictive value. The reason is that clinically apparent recurrent venous thromboembolism in untreated false-negative patients is a low-frequency outcome, approximating $10 \%$ or less (12). Accordingly, the true false-negative rate of scintigraphy likely is greater than that measured by clinical outcome.

The study of Glaser et al. does not specify how (or whether) clinical assessment and D-dimer measurement were used in evaluating their patients. This information is important to assessing their results by defining the spectrum of disease in their patients. If their referring clinicians missed the opportunity to exclude PE without using imaging at all - thus further reducing cost and radiation exposure (13) - then Glaser et al. studied a sample with excess negative cases, which would flatter the negative predictive value of scintigraphy. If this is the case, then implementing a clinical pathway that includes appropriate use of a clinical 
prediction rule and D-dimer should be the next step in their collaboration with their referring colleagues.

We also suggest that they should go further in developing clinical use of scintigraphy. We have found $(8,10)$ that perfusion scintigraphy alone can provide excellent sensitivity and specificity, with nondiagnostic rates as low as zero. Eliminating the ventilation scintigram reduces cost, further reduces radiation dose, and makes scintigraphy easier to provide on call by reducing technical complexity. Some have advocated broader adoption of the PISAPED criteria (14), albeit in a rather confused version. Our experience convinces us of the validity of the PISAPED results. However, published validation of PISAPED has been limited thus far to the PISAPED group and its collaborators. Without independent confirmation, it is not scientifically correct to recommend a substantial change (particularly in the United States) of clinical practice. The Montefiore group has the expertise and the clinical material to perform an independent evaluation of the PISAPED approach, and we urge them to do so.

An important but generally ignored phenomenon is that the most-cited studies of PE diagnosis around the world have evolved progressively from including a broad spectrum of patients to focusing primarily on convenience samples of outpatients. Space limitations do not permit a comprehensive list of references to support this assertion, but for example, in PIOPED (4) $68 \%$ of the patients studied were inpatients, including many intensive care unit patients, whereas in PIOPED II (15) only $8 \%$ of patients were inpatients and in PIOPED III (16) only 9\% of patients were inpatients, with $0 \%$ intensive care unit patients. In addition, the prevalence of PE in many recent study samples has decreased. For example, in PIOPED (4) it was 33\%, whereas in PIOPED II (15) it was $23 \%$ and in the study of Glaser et al. it was less than $10 \%$. A $2 \times 2$ table simulation demonstrates that, for a test with $90 \%$ sensitivity and $95 \%$ specific- ity, the positive predictive value is $90 \%$ with $30 \%$ prevalence of disease, but only $67 \%$ with $10 \%$ prevalence. The characteristics of the patients in different studies may also influence the sensitivity and specificity of a diagnostic test. Thus, it may be difficult to compare the performance of the test in different populations. Three conclusions follow from these observations, which apply both to scintigraphy and to CT pulmonary angiography. First, reduction in positive predictive value could result in unnecessary anticoagulation in a significant number of patients. Only patients with negative results are considered in outcome studiesusually, no research follow-up is performed of patients with a positive study. Follow-up perfusion scintigraphy in patients diagnosed with $\mathrm{PE}$ could be of great clinical utility to exclude false-positive diagnosis; repeated CT pulmonary angiography would be less acceptable because of radiation dose. Second, one must be cautious when comparing the results of older and more recent studies of PE diagnosis. It is likely that we are producing increasingly precise (and pleasing) answers to increasingly narrow (and easy) questions. Third, we need to recognize that we probably do not know with precision the accuracy of scintigraphy or CT pulmonary angiography for evaluating inpatients-in particular, critically ill inpatients. Accordingly, it would be helpful to know the demographics of the patient sample of Glaser et al.

Considering the relatively low mean age $(\approx 50 \mathrm{y})$ and the prevalence of women $(73 \%)$ among the patients in the study of Glaser et al., the scintigraphic strategy resulted in an important reduction of radiation dose, particularly for the female breast. Their demonstration of good outcomes in the pediatric population with scintigraphy is also important in this regard.

The importance of making scintigraphy available in a manner that meets the needs of clinical workflow is highlighted in the article of Glaser et al. Introduction of the trinary system did not affect substantially the number of scintigrams performed or patient outcomes, so although it is an advance, it appears less influential than test availability to adoption of scintigraphy by referring physicians. Lack of clinically relevant availability of scintigraphy is usual in most centers and is an enormous deterrent to its clinical use. The Montefiore group has demonstrated that scintigraphy can be available effectively on a sustained basis, with a significant impact on patient care (17).

Conclusions from other recent data are supported and extended by the important work of Glaser et al.: scintigraphy is an excellent diagnostic test for PE when used correctly, available conveniently in the clinical setting, and interpreted by competent, confident readers. In other words: you can't always get what you want, but if you try sometimes you just might find you get what you need.

\section{ACKNOWLEDGMENT}

No potential conflict of interest relevant to this article was reported.

\section{Henry Dirk Sostman}

Methodist Hospital

Weill Cornell Medical College

Houston, Texas

\section{Massimo Pistolesi}

University of Florence

Florence, Italy

\section{REFERENCES}

1. Glaser JE, Chamarthy M, Haramati LB, Esses D, Freeman LM. Successful and safe implementation of a trinary interpretation and reporting strategy for V/Q lung scintigraphy. J Nucl Med. 2011;52:15081512.

2. Bossuyt PM, Reitsma JB. Standards for reporting of diagnostic accuracy: the STARD initiative. Lancet. 2003;361:71.

3. Gray HW, McKillop JH, Bessent RG. Lung scan reporting language: what does it mean? Nucl Med Commun. 1993;14:1084-1087.

4. The PIOPED Investigators. Value of the ventilation/perfusion scan in acute pulmonary embolism. JAMA. 1990;263:2753-2759.

5. Stein PD, Gottschalk A. Review of criteria appropriate for a very low probability of pulmonary embolism on ventilation-perfusion lung scans: a position paper. Radiographics. 2000;20:99-105.

6. Gottschalk A, Stein PD, Sostman HD, Matta F, Beemath A. Very low probability interpretation 
of V/Q lung scans in combination with low probability objective clinical assessment reliably excludes pulmonary embolism: data from PIOPED II. J Nucl Med. 2007;48:1411-1415.

7. Stein PD, Gottschalk A, Henry JW, Shivkumar K Stratification of patients according to prior cardiopulmonary disease and probability assessment based on the number of mismatched segmental equivalent perfusion defects. Chest. 1993;104:1461-1467.

8. Miniati M, Pistolesi M, Marini C, et al. Value of the perfusion lung scan in the diagnosis of pulmonary embolism: results of the prospective investigative study of acute pulmonary embolism diagnosis. Am J Respir Crit Care Med. 1996;154:1387-1393.

9. Sostman HD, Stein PD, Gottschalk A, Matta F, Hull R, Goodman L. Acute pulmonary embolism: sensitivity and specificity of ventilation-perfusion scintigraphy in PIOPED II study. Radiology. 2008;246:941-946.

10. Sostman HD, Miniati M, Gottschalk A, Matta F, Stein PD, Pistolesi M. Sensitivity and specificity of perfusion scintigraphy combined with chest radiography for acute pulmonary embolism in PIOPED II. J Nucl Med. 2008;49:1741-1748.

11. Anderson DR, Kahn SR, Rodger MA, et al. Computed tomographic pulmonary angiography vs ventilation-perfusion scintigraphy in patients with suspected pulmonary embolism. JAMA. 2007;298:2743-2753.

12. Stein PD, Henry JW, Relyea B. Untreated patients with pulmonary embolism: outcome, clinical and laboratory assessment. Chest. 1995;107:931-935.
13. Agnelli G, Becattini C. Acute pulmonary embolism. $N$ Engl J Med. 2010;363:266-274.

14. Reinartz P. To PIOPED or not to PIOPED. J Nucl Med. 2008;49:1739-1740.

15. Stein PD, Fowler SE, Goodman LR, et al. Multidetector computed tomography for acute pulmonary embolism. N Engl J Med. 2006;354: 2317-2327.

16. Stein PD, Chenevert TL, Fowler SE, et al. Gadolinium-enhanced magnetic resonance angiography for pulmonary embolism. Ann Intern Med. 2010;152:434-443.

17. Stein EG, Haramati LB, Chamarthy M, et al. Success of a safe and simple algorithm to reduce use of CT pulmonary angiography in the emergency department. AJR. 2010;194:392-397. 